\title{
Marie de Gournay ou l'occultation d'une figure auctoriale
}

\author{
JEAN-PHILIPPE \\ BEAULIEU
}

Summary: Beginning with the recognition that the records and biographical dictionaries of the seventeenth and eighteenth centuries devote greater attention to the personal aspects of Marie de Gournay (1565-1645) than to her literary production, which was nonetheless substantial, this study considers the forms of, and reasons for, such an obscuring of her authorial standing. Among the probable explanations, what stands out is the less than enthusiastic reception reserved by the seventeenth century for the intellectual ambitions evident in that "women's writing" with which Gournay seems to have established so close a connection as finally to transform her work into a textual alter ego of the author.

$\mathrm{B}^{\text {ien que Marie le Jars de Gournay (1565-1645) soit l'auteur d'un grand }}$ $\mathrm{B}_{\text {nombre d'écrits, les documents ou dictionnaires biographiques des XVII }}$ et XVIII siècles passent souvent sous silence cet ensemble textuel considérable, pour ne retenir que la remarquable érudition de la « fille d'alliance » de Montaigne. Cette façon dont les rédacteurs de notices biographiques semblent méconnaître les réalisations de Gournay au profit de ses seules dispositions intellectuelles n'est certainement pas étrangère à la position en porte-à-faux qui a caractérisé certains des rapports de Marie le Jars avec les institutions et courants littéraires de son époque ${ }^{1}$. S'il est indéniable, comme elle le dit elle-même, que ses textes sont porteurs de « sentiments moulés à l'air d'un autre Siecle »², c'est-à-dire celui de l'humanisme, j'aimerais suggérer qu'une telle occultation de la production textuelle de Gournay trouve son origine moins dans la résistance aux transformations culturelles de la première moitié du XVII ${ }^{\mathrm{e}}$ siècle qui lui est souvent attribuée ${ }^{3}$, que dans la nature même des activités d'écriture auxquelles elle s'est vouée et dont le caractère singulier ne pouvait que heurter la sensibilité 
de ses contemporains. En effet, mettant de côté les occupations qui définissent et circonscrivent traditionnellement le rôle social de la femme, Gournay a tourné tous ses efforts vers l'élaboration d'une œuvre textuelle ambitieuse et savante, fort éloignée des passe-temps bienséants auxquels était tenue de se livrer l'« honneste femme » comme l'a représentée Du Boscq en 16394. Ne serait-ce que par son ampleur et sa diversité, la production de Gournay témoigne d'un constant investissement de l'écriture par des préoccupations intellectuelles, lequel a résulté, à la suite d'un travail attentif de révision et de réécriture, en un ensemble de près de mille pages dans ses Advis de 1641, regroupant des textes variés touchant aussi bien des questions de poétique et de traduction, que d'éducation et de morale. De plus, l'écriture gournayenne affirme un rapport très étroit au texte qui fait de celui-ci l'alter ego de l'auteure, c'est-à-dire l'expression de sa pensée et le prolongement de sa voix. Ce lien intime entre Gournay et son texte est souligné non seulement par les pièces liminaires de son recueil d'Advis de 1641, mais, de façon plus générale, par l'orientation énonciative de l'ensemble du livre, qui, inspirée de l'exemple montaignien, place constamment la figure auctoriale de Gournay au cœur même de son œuvre, en faisant de cette figure le point de convergence et le facteur d'unité de l'ouvrage. Cette manière de faire corps avec son texte relève d'une entreprise d'écriture ambitieuse, et à certains égards moderne ${ }^{5}$, qui suggère que l'inscription de cette femme de lettres dans les changements culturels de son époque ne se réduit pas à la position passéiste qui lui est souvent associée. Dans le cadre de cette étude, je chercherai d'abord à rendre compte de l'occultation de la figure auctoriale de Gournay qui caractérise l'ensemble des documents d'Ancien Régime, pour, par la suite, tenter d'expliquer certaines modalités de cette occultation à la lumière du lien singulier établi par Gournay avec son œuvre.

Peut-être en raison de ses rapports avec celui qu'elle appelait son « second père », Gournay fait partie de ces femmes écrivains de l'Ancien Régime qui ont échappé à l'oubli après leur mort ${ }^{6}$. En effet, certaines listes de femmes célèbres publiées au XVII ${ }^{\mathrm{e}}$ siècle, de même que plusieurs dictionnaires biographiques ultérieurs, lui consacrent des notices substantielles ; c'est le cas du New and General Biographical Dictionary de 1784, qui lui accorde même un article sensiblement plus long que celui portant sur $\mathbf{M}^{\mathrm{me}}$ de Sévigné ${ }^{7}$. Notons que, dans l'ensemble de ces notices, les activités d'écriture de Gournay retiennent très peu l'attention des rédacteurs. C'est probablement un trait des genres biographiques - surtout lorsqu'il est question d'une femme - d'obliger le lecteur à se frayer un chemin à travers une dense couche anecdotique avant d'entrapercevoir les textes eux-mêmes, le plus souvent relégués à l'arrière-plan. C'est particulièrement vrai pour 
Marie de Gournay, qui arrive difficilement à s'imposer comme figure auctoriale dans les documents des XVII ${ }^{\mathrm{e}}$ et XVIII ${ }^{\mathrm{e}}$ siècles.

Si la soif de savoir et les moyens intellectuels de Gournay ont souvent été notés positivement par ses contemporains, les enjeux et la portée de sa production textuelle semblent les avoir assez peu intéressés. Dans Les Eloges et les vies des Reynes, des princesses et des dames illustres en pieté, publiés deux ans après la mort de Gournay et qui ont donc valeur de témoignage contemporain, Hilarion de Coste juge cette « docte fille [...] capable de donner l'envie aux plus doctes de tous les hommes », et signale qu'elle «passoit les jours \& les nuits en l'étude des belles sciences $»^{8}$. Pourtant, il ne consacre à ses recueils que trois lignes sur un total de cinq pages, se contentant d'en mentionner le titre ${ }^{9}$. Dans son ouvrage polémique intitulé Les Dames illustres (1665), Jacquette Guillaume ne parle aucunement des écrits de Gournay, mais rapporte que, selon Juste Lipse, celle-ci « estoit en si grande reputation d'esprit, d'eloquence et de doctrine, qu'elle pouvoit fermer la bouche aux plus sçavans hommes de ce siecle ${ }^{10}$. En 1663 , dans Le Cercle des femmes sçavantes, Jean de La Forge fait également un éloge de Marie de Gournay mentionnant son «beau Livre d'advis » pour insister surtout, de manière elliptique, sur l'étendue de son savoir : «Je croirois faire tort à toutes les personnes qui se meslent du commerce des Sciences, si je pretendois leur apprendre quelle est cette celebre Fille ${ }^{11}$. La Forge est curieusement plus loquace au sujet des textes de Marie de Romieu et de Suzanne de Nervèze ; pourtant, on aurait pu s'attendre au contraire... Dans De la connoissance des bons livres, Charles Sorel s'attache, comme ses prédécesseurs, à souligner la générosité et les autres vertus de Gournay $^{12}$; dans sa Bibliothèque françoise, il laisse cependant entrevoir l'ambition de l'entreprise d'écriture gournayenne, par la mention plutôt élogieuse de certains traités éducatifs et poétiques, bien que ce soit essentiellement pour montrer « combien cette illustre fille avoit l'esprit ferme \& genereux ${ }^{13}$. Si, dans les documents des XVII ${ }^{\mathrm{e}}$ et XVIII ${ }^{\mathrm{e}}$ siècles, on ne retient souvent des activités d'écriture de Marie de Gournay que les différentes versions de sa préface aux Essais de Montaigne ${ }^{14}$, il est clair, pourtant, lorsqu'on examine l'ensemble textuel qu'elle a laissé, que toute son activité est tributaire d'une volonté de contribuer au savoir et de laisser sa marque par l'écriture. C'est ce que souligne l'Apologie pour celle qui escrit, texte à caractère autobiographique qui conclut le premier livre des Advis, et dont il est loisible d'interpréter le titre dans le sens d'une définition sociale de soi passant nécessairement par l'activité scripturale. En effet, dans cette formule apparemment banale se trouve exprimé - par un simple principe de contiguïté - le lien intime qui unit l'écriture et sa justification dans l'affirmation identitaire de Gournay, désignée non seulement comme celle qui écrit ce 
texte en particulier, mais aussi, de manière intransitive, comme celle qui écrit tout court ${ }^{15}$.

Inspiré en partie par les modèles humanistes qui ont marqué sa jeunesse, le projet qui paraît avoir animé Gournay la majeure partie de sa vie semble moins convenable au siècle suivant ${ }^{16}$, au moment où l'érudition, et tout particulièrement son étalage, sont modalisés par une vision de la bienséance plus étroite, surtout en ce qui a trait au savoir féminin ${ }^{17}$. C'est vraisemblablement le désir de Gournay de se définir essentiellement par son œuvre qui lui a valu l'incompréhension de plusieurs de ses contemporains, peu disposés à admettre, chez une femme, le caractère aussi entier d'une telle présence au monde ${ }^{18}$. D'où le foisonnement de l'aspect anecdotique qui, dans bon nombre de notices, masque la figure d'écrivain de Gournay par une insistance sur les traits - ou, à vrai dire, sur l'absence des traits — qui désignent positivement le sexe féminin : beauté, charme, esprit de finesse. On passe ainsi plus de temps à parler de Gournay (et de ses travers) que de son œuvre. Si l'on s'attarde à tracer le portrait d'une « femme savante », c'est en insistant sur les dispositions de celle-ci plutôt que sur ses réalisations. Ainsi, dans le dictionnaire anglais mentionné plus haut, on signale de façon positive le respect que lui valait son érudition ${ }^{19}$. Mais cette érudition peut rapidement se transformer en objet de ridicule, lorsqu'on juge qu'elle n'est pas accompagnée des attributs féminins habituellement valorisés. Par exemple, dans le Dictionnaire des portraits historiques de 1773, on concède que « les langues savantes lui étoient familieres » et qu'elle «écrivit dans la sienne mieux qu'aucune femme de son temps », mais on finit toutefois par la décrire comme

une espèce de prude, une femme philosophe qui avoit tourné toutes ses études du côté de la morale et du raisonnement. Elle n'ignoroit pas que les talents agréables conduisent rarement une femme à l'immortalité, lorsqu'une figure charmante ne la fait pas valoir, et Mlle de Gournay n'était nullement jolie ${ }^{20}$.

Cette façon d'invalider la valeur intellectuelle par le recours à des considérations d'ordre physique suggère que, si Gournay s'est adonnée à des activités de l'esprit, c'est qu'elle était dépourvue de charmes féminins ${ }^{21}$. À peine mentionnée, l'œuvre textuelle se présente au mieux comme un pis-aller, comme une façon de s'occuper lorsqu'on ne peut faire mieux. Laisser ainsi apparaître comme dérisoires les ambitions féminines qui ne sont pas contenues dans les limites des attentes sociales relève de ce que l'on peut appeler le syndrome du bas-bleu, stigmatisation d'une figure du savoir féminin que Gournay a incarnée pour plusieurs générations de lettrés 22 .

Il y a lieu de se demander si cette manière de mettre la personne au premier plan ne relève pas en grande partie d'un malaise éprouvé face à 
l'ambition gournayenne non seulement de «faire œuvre », mais d'assurer la portée et la pérennité de sa production textuelle. Le fait de privilégier le discours savant (le «Discours de raison », comme le nomme Gournay [Advis, f. br]) et de lui donner une orientation souvent polémique, de même que la volonté de présenter le fruit de son travail sous la forme d'un ensemble monumental, correspondent assez peu à l'horizon d'attente des années 1630 et 1640 . C'est justement l'écart entre cette entreprise et sa réception qui explique la récurrence des «propos désenchantés » que tient Gournay ${ }^{23}$, expression de la distance qui existe, dans la perception de celle-ci, entre la réalisation d'un « Ouvrage purement intellectuel » (Advis, f. aiij $\left.{ }^{\mathrm{r}}\right)$ et l'accueil réservé à ce dernier en un siècle qu'elle juge « insolent \& coursaire » (Advis, p. 107), où « se voyent communément [...] le mespris ou l'emploite impertinente des Lettres » (p. 8). D'où les affirmations qui, à la manière d'un défi, expriment le désir de poursuivre son œuvre et de l'offrir au public en dépit de l'appréhension d'un accueil peu bienveillant : «mon Livre n'espere pas de rencontrer une oreille favorable », dit-elle à ce propos dans l'avis $A u$ lecteur (Advis, f. cij ${ }^{\mathrm{r}}$ ). À une époque où l'« on fagotte à fantasie l'image des femmes Lettrées », l'ambition de Gournay « d'estre clair-voyante \& sçavante, ou d'avoir [...] desiré de se rendre telle » (Advis, p. 605[B]) la place dans une position qui semble mise sous le signe de l'excès - du trop ou du trop peu - , dont elle peut difficilement sortir gagnante. Marie de Gournay elle-même souligne la conscience qu'elle a de la double contrainte de cette position, lorsqu'elle affirme que « tous les jours [son] ignorance en sert de risée aux esprits guaillards d'entre les sçavans, comme [sa] Science en sert aux autres » (ibid.). Ainsi, comme le laisse entendre l'extrait du dictionnaire de 1773 cité plus haut, même l'érudition que certains contemporains de Gournay admirent chez elle semble être porteuse d'une connotation liée à l'excès, propension qui est tacitement associée à sa personne même. Michel de Marolles, dans ses Mémoires, souligne qu'elle « savoit force choses qui ne sont pas ordinaires aux personnes de son sexe $»^{24}$. Pourtant, on sent se profiler chez lui un jugement implicite sur l'« ame candide et genereuse » de Marie de Gournay, jugement que la postérité accentuera au point de devenir caricatural, en rapportant à la suite de Tallemant des Réaux des anecdotes qui soulignent la naïveté et l'emportement de celle que l'on pouvait facilement abuser en flattant son amour-propre ${ }^{25}$. De la même façon, Charles Sorel juge utile de conclure son commentaire sur Gournay par une référence à son caractère colérique : «[...] ceux qui l'on veuë autrefois sçavent qu'elle avoit des emportements horribles ${ }^{26}$. Par-delà ce que qu'elles paraissent révéler quant à la personnalité de Gournay, ce qui frappe dans ces anecdotes — et dans l'exploitation caricaturale que la postérité en a fait ${ }^{27}$ — , est la façon de constamment ramener au premier plan le caractère excessif d'une 
figure féminine dont le projet de vie tend à se confondre avec le projet d'écriture. Déjà, le désir gournayen de vivre de sa plume demeure exceptionnel pour une femme de l'époque. Mais que dire si, comme Marie de Gournay, on se définit essentiellement par cette plume, au point de rendre indistinctes les frontières entre ses idées et le texte qui leur sert de véhicule ? Ce dernier est systématiquement lié à des procédés d'affirmation de l'identité de Gournay en tant qu'auteur, procédés qui désignent le texte comme un alter ego, dans les termes d'un prolongement ou d'une projection de soi-même. Le titre attribué par Gournay au recueil de 1626, L'Ombre de la Damoiselle de Gournay, présente d'emblée son œuvre comme un ensemble dont l'appellation doit se comprendre, d'après l'explication qu'elle fournit, comme « l'Image de [son] esprit, maistresse piece de [son] estre » (Advis, f. biij $\left.{ }^{\mathrm{v}}\right)$. Ainsi que le suggère l'inscription latine de la page de titre de 1626 ( «Pactura nepotibus umbram »), cette image vise, à la manière du sapin qui orne la page en question, à couvrir de son ombre les générations à venir. Le lien qui s'établit ici entre le texte et l'auteure est des plus étroits, le texte devenant la projection platonicienne ou, si l'on préfère, la matérialisation intellectuelle de l'esprit de l'auteure. Il est intéressant de noter que cette formule au singulier, qui semble avoir déplu à certains, est remplacé en 1634 et 1641 par un intitulé au pluriel, faisant du recueil — conçu initialement sur le mode intangible d'une projection de l'auteure - un objet plus facile à cerner et à soumettre à la dynamique des échanges commerciaux. Dépouillée du caractère entier et étroitement identitaire du titre originel, l'œuvre se réduit plus modestement à une offrande de textes faite au public. Dans le Discours sur ce Livre de 1641, Gournay déplore d'ailleurs ce changement, en le présentant comme une nécessité de librairie à laquelle elle a dû se plier : " [Ce livre] s'appelleroit toujours [...] L'Ombre de la Damoiselle de Gournay [...] s'il ne falloit contenter mon Libraire qui craint, ce semble, les Esprits. Je suis trompée si cét ancien nom de mes chetives Oeuvres, n'est preferable au nouveau, quoy qu'ayent voulu dire certains Critiques de nostre saison » $(\text { Advis, f. biij })^{28}$.

Une autre manifestation du rapport étroit de Gournay avec son œuvre est l'utilisation de la métaphore de la maternité. À quelques reprises dans les pièces liminaires, l'auteure se présente comme la mère de ses écrits. Dans le Discours sur ce Livre, par exemple, elle invite la dédicataire, Sophrosyne, à passer de «l'examen du Livre à celuy de la foy de sa mere » (Advis, f. biij $)$. Cette image, qui lie analogiquement responsabilités parentale et auctoriale, n'est pas neuve et certainement pas assez prégnante dans le texte pour être significative à elle seule, mais elle participe au système énonciatif qui rend tangible la voix auctoriale. Dans bon nombre des formes textuelles que privilégient les Advis, on retrouve des modalités énonciatives où se cristal- 
lise l'expression du «je » auctorial. Ainsi, parmi les quelque cinquante-trois textes dont le titre figure dans la table des matières, tous ou presque participent à un degré ou à un autre d'un mode énonciatif faisant entendre la voix de l'auteure et dessinant, comme le suggère Anna Lia Franchetti, «le portrait d'un scripteur en mouvement, qui crée et se crée à travers sa "prose discourante" ${ }^{29}$. Il s'agit là d'un facteur d'unification du recueil qui est renforcé par le grand nombre de renvois que Gournay effectue à ses autres textes. Ces renvois, explicites ( «comme j'ay dit au traicté des Metaphores » [p. 440]) ou allusifs (« comme je remarquois ailleurs » [p. 249]), relèvent rarement d'une stricte finalité référentielle. À vrai dire, par une insistance sur l'activité scripturale, ils servent avant tout à tisser un réseau de rappels de la fonction auctoriale ; réseau qui laisse entendre que ce livre de «meslanges » ne répond pas à un simple principe de cumul aléatoire, mais que les différents aspects de sa réalisation font l'objet d'une prise en charge qui culmine dans la responsabilité éditoriale que l'auteure a assumée dans sa révision et sa parution. Il y a tout lieu de croire, en effet, que Gournay a été attentive à la production de ses recueils, surtout celui de 1641, dont elle semble avoir revu les épreuves en cours d'impression, comme le suggère, dans certains exemplaires de cette édition, la présence de deux listes d'errata : la première intégrée au texte, la seconde ajoutée après coup. De plus, dans cette deuxième liste, la voix de l'auteure se fait distinctement entendre, réactivant le topos de la maternité, de manière à assumer l'ultime responsabilité de la correction matérielle de son texte, qu'elle doit finalement abandonner à son sort, non sans hésitation : «Permets, Lecteur, ce dernier soin à une pauvre mère preste à quitter son enfant orphelin, \& veuf de toute assistance. Je te recommande ce qui peut estre encore eschappé à ma derniere recherche $»^{30}$.

Gournay prend donc en charge son œuvre à diverses étapes de sa réalisation. Cette prise en charge revêt un caractère défensif qui donne à plusieurs textes gournayens leur tonalité polémique caractéristique. La présence même de nombreux commentaires, qui rendent compte du positionnement de Gournay face à certaines questions et à certains interlocuteurs, souligne avant tout son intention de participer à des débats qui l'engagent entièrement en tant qu'auteur (au sens d'autorité). Sur ce plan, encore, elle se montre soucieuse de faire corps avec des idées dont elle prend systématiquement la défense, au moyen de stratégies de réponse qui mettent en relief le soin avec lequel elle cherche à établir un dialogue aussi bien avec ses dédicataires et ses protecteurs qu'avec ses détracteurs. Dans ce dialogue qui s'inscrit dans la diachronie - puisqu'il entraîne des modifications parfois très substantielles d'une version à l'autre du recueil ${ }^{31} \ldots$, s'affirme le « je » gournayen qui, par-delà la diversité des matières traitées, donne son 
unité principale au recueil. Les affirmations de Gournay relatives à la « deffaveur où [elle] vi[t] en [son] siècle », tout en révélant un refus d'adhérer à certaines tendances esthétiques et intellectuelles de la première moitié du XVII ${ }^{e}$ siècle, témoignent néanmoins - et surtout — d'une volonté de participer à la vie culturelle et d'y faire entendre sa voix. Il ne faudrait pas souscrire trop hâtivement à l'image d'une Gournay isolée et en marge des courants du XVII e siècle. Ses contacts avec des savants et des personnalités en vue étaient loin d'être négligeables ${ }^{32}$. Marolles mentionne Boisrobert, Colletet, Malleville, La Mothe Le Vayer ${ }^{33}$. Hilarion de Coste fait état d'une correspondance avec de nombreuses personnalités : Du Perron et Richelieu, François de Sales, Juste Lipse, Daniel Heinsius, Anna Maria van Schurman et bien d'autres ${ }^{34}$. Au début du XIX ${ }^{\mathrm{e}}$ siècle, Fortunée Briquet affirme quant à elle que la comtesse de Soissons, la duchesse de Longueville et la princesse de Gonzague-Nevers « aimaient à s'instruire dans sa conversation » ${ }^{35}$. Les dédicaces des textes (surtout des poèmes de son Bouquet de Pinde) indiquent non seulement la nécessité de s'assurer le patronage de personnages en vue, mais aussi l'inscription de Marie de Gournay dans certains réseaux sociaux et intellectuels de son époque ${ }^{36}$.

La dynamique même des textes suggère donc que Gournay n'est pas restée indifférente aux transformations culturelles qu'a connues son époque. Et, si ses positions sur plusieurs questions, notamment poétiques et stylistiques, révèlent un attachement à l'héritage du siècle précédent, il y a d'autant moins de raison de cantonner Gournay à une simple position de repli que, comme le suggère Dominique de Courcelles, elle fait preuve d'un esprit de dérision, voire même d'autodérision ${ }^{37}$, qui rend l'orientation de ses propos beaucoup plus dynamique et nuancée qu'on ne le croit généralement ${ }^{38}$. Ainsi l'ironie de Gournay colore-t-elle une volonté polémique d'autojustification, qui la montre attentive au présent et soucieuse d'affirmer la singularité de son traitement de certaines questions. Il n'est pas indifférent à cet égard qu'une bonne partie du Discours sur ce Livre - véritable guide de lecture du recueil - cherche à préciser le statut de chacun des textes relativement à leur « originalité », critère qui devient l'aune servant à mesurer la valeur de l'œuvre :

Il faut voir en premier lieu si [le corps de l'œuvre] se pare de cette qualité qui proprement le fait Livre, de se pouvoir nommer Original, puisque ceux qui ne peuvent porter ce tiltre pour estre empruntez d'autruy, sont à bien parler les enfans de Livres seulement : car pour qu'une CEuvre mérite ce nom de Livre, il faut qu'elle apporte au Public des biens propres \& nouveaux, nez en l'esprit de leur Autheur. (Advis, f. $\mathrm{b}^{\mathrm{r}}$ ) 
Bien sûr, le qualificatif « original » ne correspond pas ici au sens moderne désignant ce qui est inédit et personnel. Gournay elle-même précise qu'elle appelle « Livre original, non pas celuy qui l'est entièrement, pource qu'il ne s'en trouveroit point de cette marque, mais bien, celuy qui l'est pour la pluspart de son estenduë » (ibid.). C'est donc moins la nouveauté absolue qui compte ici que la possibilité de laisser sa marque, de se distinguer en contribuant à une réflexion (« d'apporter des biens propres et nouveaux »), de façon à devenir un modèle, une nouvelle origine, selon l'une des acceptions du terme « original » courante au XVII ${ }^{\mathrm{e}}$ siècle $^{39}$. Si le sens du mot n'est donc pas inhabituel, l'insistance avec lequel il est ici utilisé est surprenante. On doit probablement y voir l'expression d'une forme de réflexivité sur sa propre écriture qui peut être considérée comme un signe auctorial d'affirmation de soi $^{40}$ et, du même coup, un signe d'émancipation de l'influence montaignienne ${ }^{41}$. En soulignant systématiquement, et par le biais de la notion d'originalité, le lien entre son livre et elle, Gournay revendique un statut, celui d'« Autheur » ${ }^{42}$, soucieux de l'intégrité d'une œuvre constamment menacée par le jugement d'autrui, comme en particulier celui de ces hommes de mauvaise foi qui s'attaquent aux ouvrages féminins :

\footnotetext{
Il sera bon de voir [...] quel Labeur de femme vainqueur de la malice des temps et des hommes, sera capable d'effacer le mien : je dy vainqueur de la malice des temps et des hommes : car quels ravages n'ont-ils point fait de cette part sur les Corinnes, les Saphons, les Hypathies, les Aretes \& autres ? (Advis, f. $\mathrm{b}^{\mathrm{r}}$ )
}

Lancée tel un défi au tout début du recueil, cette question en dit long à la fois sur les ambitions de Marie de Gournay et sur le sort incertain réservé, selon elle, à la production textuelle des femmes. Dans la mesure où Gournay tend à se définir par son œuvre, on peut saisir la nature de ses appréhensions et de ses doléances face aux aléas de la réception de celle-ci, puisque toute atteinte à ses textes représente en fin de compte une atteinte à sa personne même. Il n'y a donc pas lieu de s'étonner que le mot final des Advis consiste en une interdiction de modifier quoi que ce soit au recueil : «Si ce livre me survit, je deffends à toute personne, telle qu'elle soit, d'y adjouster, diminuer, ny changer jamais aucune chose, soit aux mots ou en la substance [...]» (Advis, p. 995). Cette interdiction trouve sa motivation moins dans la conviction que l'œuvre est parfaite et définitive que dans un sens aigu de la responsabilité auctoriale ${ }^{43}$. On peut ainsi comprendre la vitalité avec laquelle Gournay défend ses idées et ses écrits, témoignant d'une volonté de vivre dans le monde et par l'écriture dont la singularité est manifestement plus éloquente aujourd'hui qu'à l'époque.

Université de Montréal 
32 / Renaissance and Reformation / Renaissance et Réforme

\section{Notes}

1. Son opposition aux réformes malherbiennes a fait l'objet de plusieurs commentaires. À ce sujet, voir notamment Anne Uildricks, Les idées littéraires de Mlle de Gournay (Gröningen, Druk, 1962), p. 38 et suiv.

2. Marie de Gournay, Les Advis, ou, les Presens de la Demoiselle de Gournay (Paris, Jean Du Bray, 1641), f. $\mathrm{c}^{\mathrm{v}}$. Sauf indication contraire, toutes les citations proviennent de cette édition, que l'on doit considérer comme la version ultime des textes en recueil de Gournay, publiés initialement en 1626, sous le titre L'Ombre de la Damoiselle de Gournay, puis en 1634 et 1641, sous celui d'Advis.

3. Voir, par exemple, les propos de Jean Larnac dans Histoire de la littérature féminine en France (Paris, Éditions Kra, 1929), p. 70.

4. Jacques Du Boscq, L'Honneste Femme (Rouen, chez la veuve DuBosc, 1639).

5. Au sujet de la configuration «moderne » des opérations discursives de Gournay, voir l'amorce de réflexion que proposent Jean-Philippe Beaulieu et Hannah Fournier, « Le discours politique de Marie de Gournay ou la modernité d'une prise de parole », dans $E M F$ : Studies in Early Modern France, vol. III : Signs of the Early Modern, sous la dir. de David Rubin (Charlottesville, Virginie, Rockwood Press, 1997), p. 69-70.

6. Marie-Thérèse Noiset (« Marie de Gournay et la caprice des siècles », Études françaises, 29.3, 1994, p. 193) nous rappelle que, jusqu'à récemment, c'est surtout « l'association de Gournay avec Montaigne et son rôle d'éditrice des Essais » qui « semblent avoir conservé quelque notoriété à l'écrivaine ».

7. A New and General Biographical Dictionary (Londres, W. Strahan, T. Pargne et alii, 1784), vol. VI, p. 161.

8. Hilarion de Coste, Les Eloges et les vies des Reynes, des princesses et des dames illustres en pieté (Paris, S. et G. Cramoisy, 1647), tome II, p. 668-69.

9. On pourrait dire la même chose de la notice des Eloges des Illustres Sçavantes (publiés à la suite des Nouvelles Observations sur la langue Françoise (Paris, Jean Cusson, 1668) calquée sur celle d'Hilarion de Coste — , où Marguerite Buffet se contente d'affirmer que Gournay « a sçavamment écrit en prose \& en vers » (p. 291).

10. Jacquette Guillaume, Les Dames illustres où par bonnes \& fortes raisons, il se prouve, que le Sexe Feminin surpasse en toute sorte de genres le Sexe Masculin (Paris, Thomas Jolly, 1665), p. 292.

11. Jean de La Forge, Le Cercle des femmes sçavantes (Paris, J.-B. Loyson, 1663), f. $\mathrm{D}^{\mathrm{r}-\mathrm{v}}$.

12. Charles Sorel, De la connoissance des bons livres (1671), éd. Lucia Moretti Cenerini (Rome, Bulzoni Editore, 1974), p. 329.

13. Charles Sorel, La Bibliothèque françoise (Genève, Slatkine, 1970 [reproduction de la $2^{\mathrm{e}}$ édition, Paris, 1667]), p. 80.

14. Consulter notamment Fortunée Briquet, Dictionnaire historique et bibliographique des Françaises, et des étrangères naturalisées en France (Paris, Imprimerie de Gillé, 1804), p. 202. Encore récemment, c'est principalement ses liens avec Montaigne qui ont valu à Gournay deux ouvrages collectifs : Marie de Gournay et l'édition de 1595 des Essais de Montaigne, Actes du colloque organisé par la Société Internationale des Amis de Montaigne les 8 et 10 juin 1995, en Sorbonne, réunis par Jean-Claude Arnould (Paris, Champion, 1997); Montaigne et Marie de Gournay, Actes du Colloque international de Duke, 31 mars- $1^{\text {er }}$ avril 1995, réunis et présentés par Marcel Tetel (Paris, Champion, 1997). Si, surtout dans 
Jean-Philippe Beaulieu / Marie de Gournay ou l'occultation d'une figure auctoriale / 33

le premier ouvrage, on consacre une portion significative de la réflexion critique à l'œuvre de Gournay en elle-même, il est curieux de lire certaines affirmations, comme celle de Jean Balsamo qui, parlant des Essais comme « le livre fameux au seul culte duquel Mademoiselle de Gournay va désormais vouer ses efforts et sa vie », paraît considérer comme négligeable les textes de celle-ci, dont il dit pourtant quelques mots un peu plus loin ( « Marie de Gournay et la famille de Montaigne : les poèmes du Proumenoir et l'édition des Essais [1594-1599] », in Montaigne et Marie de Gournay, p. 187).

15. Je renvoie à la lecture de ce texte que propose Domna Stanton, « Autogynography: The Case of Marie de Gournay's Apologie pour celle qui escrit », in Autobiography in French Literature, French Literature Series 12 (Columbia, South Carolina, Department of Foreign Languages and Literatures, College of Humanities and Social Sciences, The University of South Carolina, 1985), p. 18-31.

16. Au sujet de l'édification par Gournay de son œuvre, voir l'article d'Anna Lia Franchetti, «L'Ombre et le monument : Marie de Gournay, éditrice de ses propres œuvres », in Marie de Gournay et l'édition de 1595 des Essais de Montaigne, notamment les p. 220 et suiv.

17. Linda Timmermans, L'accès des femmes à la culture (1598-1715). Un débat d'idées de saint François de Sales à la marquise de Lambert (Paris, Honoré Champion, 1993), p. $58-59$.

18. On retrouve des échos d'une telle incompréhension réprobatrice jusque dans les paroles de Larnac qui, au début du $\mathrm{XX}^{\mathrm{e}}$ siècle, affirme que Gournay « avait eu le tort de vouloir singer l'homme. Et cela, les hommes ne lui ont pas pardonné » (p. 72).

19. «She was much respected [...]. However, she did not escape the fate which usually attends eminent wits. Aspersion and abuse is a tax which has always been laid on superior excellence, and Mad. de Gournay was not without her share of these » (A New and General Biographical Dictionary, p. 161).

20. Dictionnaire des portraits historiques, avec anecdotes et traits remarquables des hommes illustres (Paris, Lacombe, 1773), vol. II, p. 94.

21. Notons que, dans son livre de 1910, Mario Schiff reconduit un tel jugement lorsqu'il affirme que « [...] Marie n'avait même pas, pour se défendre, la beauté qui fait que les hommes pardonnent aux femmes d'aimer ce qu'ils n'aiment pas » (La fille d'alliance de Montaigne, Marie de Gournay [Paris, Slatkine, 1978 (reproduction de l'édition de Paris, Champion, 1910)], p. 37).

22. «[...] elle eut le tort de ne voir la vie qu'à travers les livres et de verser dans la pédanterie », affirme Jean Larnac (p. 69).

23. Pour reprendre les termes de Marie-Thérèse Noiset (p. 195).

24. Michel de Marolles, Mémoires (Amsterdam, s.é., 1755), vol. I, p. 110.

25. Gédéon Tallemant des Réaux, Historiettes, éd. Antoine Adam, coll. «Bibliothèque de la Pléiade » (Paris, Gallimard, 1960), vol. I, p. 382-84. Dans son Apologie, Gournay ellemême se défend des excès qu'on lui a reprochés, principalement d'avoir dilapidé ses avoirs pour payer des expériences d'alchimie : « Je diray donc [...] que ma mauvaise fortune ne vient pas de l'alchimie, ny d'autre mien excés » (Advis, p. 615).

26. Sorel, De la connoissance des bons livres, p. 329.

27. Depuis plusieurs années, certains commentateurs ont cherché à réévaluer cette façon de représenter Gournay et son œuvre. Récemment, Giovanni Dotoli a affirmé de nouveau le caractère impératif d'une telle réévaluation, en proposant un bref survol de la littérature 
34 / Renaissance and Reformation / Renaissance et Réforme

critique déjà engagée dans cette voie (« Montaigne et les libertins via Mlle de Gournay », in Montaigne et Marie de Gournay, p. 126-27).

28. Au sujet du titre du recueil, voir le commentaire de Géralde Nakam, « Marie le Jars de Gournay, "fille d'alliance" de Montaigne (1565-1645) », in Marie de Gournay et l'édition de 1595 des Essais de Montaigne, p. 20-21.

29. Franchetti, p. 220.

30. Pour une retranscription de cette seconde liste, consulter l'édition des Advis de 1641 préparée par Jean-Philippe Beaulieu et Hannah Fournier, vol. I (Amsterdam, Rodopi, 1997), p. 64.

31. Consulter Jean-Philippe Beaulieu et Hannah Fournier, « Pratiques dialogiques et réécriture dans l'œuvre de Marie de Gournay », Neophilologus, 82 (1998), p. 359-62.

32. Constant Venesoen, Études sur la littérature féminine au XVIIe siècle (Birmingham, Alabama, Summa Publications, 1990), p. 13.

33. Marolles, vol. I, p. 111-12.

34. Hilarion de Coste, tome II, p. 669.

35. Dictionnaire, vol. II, p. 203.

36. Voir Noiset, p. 195-96.

37. C'est le cas de la première liste d'errata de l'édition de 1641 qui présente ironiquement le profil de l'auteure comme celui « d'une pauvre vieille, qui corrigeant son propre Ouvrage sur la presse, croit tousjours lire ce qu'elle a escrit».

38. Dominique de Courcelles, « Le rire de Marie de Gournay, fille d'alliance de Montaigne », in Montaigne et Marie de Gournay, p. 228 et suiv.

39. Gaston Cayrou, Le français classique. Lexique de la langue du dix-septième siècle (Paris, Didier, 1948), p. 628.

40. Pour un examen de certaines des marques de réflexivité chez Gournay, voir Marie-Thérèse Noiset, « Le jeu du moi dans les visées politiques et morales de Marie de Gournay », in Dans les miroirs de l'écriture. La réflexivité chez les femmes écrivains d'Ancien Régime, sous la dir. de Jean-Philippe Beaulieu et Diane Desrosiers-Bonin, coll. « Paragraphes » (Montréal, Dép. d'Études françaises de l'Université de Montréal, 1998), p. 108-16.

41. À ce sujet, consulter Évelyne Berriot-Salvadore, «L'héritage de Montaigne ou les voies de l'émancipation de Marie de Montaigne », in Marie de Gournay et l'édition de 1595 des Essais de Montaigne, p. 154-60.

42. Ou même celui d'« Authrice», pour reprendre une forme féminine qu'utilise Gournay.

43. Ce qui ne constitue pas pour autant un aveu de l'impossibilité de parfaire le monument que, selon Anna Franchetti (p. 220-22), Gournay cherche à édifier. Peut-être l'idée de la « recherche obstinée d'une forme accomplie » (p. 232) ne rend-elle compte que partiellement d'une dynamique d'écriture qui en vient à assumer — et peut-être même à défendre - sa propre imperfection. 